\title{
A new determination of the extragalactic background of diffuse gamma rays taking into account dark matter annihilation
}

\author{
W. de Boer, A. Nordt, C. Sander, and V. Zhukov \\ Institut für Experimentelle Kernphysik, Universität Karlsruhe (TH), PO Box 6980, 76128 Karlsruhe, Germany \\ e-mail: Wim.de.Boer@cern.ch, [sander; zhukov]@ekp.uni-karlsruhe.de \\ Received 30 November 2006 / Accepted 2 May 2007

\section{ABSTRACT}

\begin{abstract}
The extragalactic background (EGB) of diffuse gamma rays can be determined by subtracting the Galactic contribution from the data. This requires a Galactic model (GM) and we include for the first time the contribution of dark matter annihilation (DMA), which was previously proposed as an explanation for the EGRET excess of diffuse Galactic gamma rays above $1 \mathrm{GeV}$. In this paper it is shown that the newly determined EGB shows a characteristic high energy bump on top of a steeply falling soft contribution. The bump is shown to be compatible with a contribution from an extragalactic DMA signal from weakly interacting massive particles (WIMPs) with a mass between 50 and $100 \mathrm{GeV}$ in agreement with the EGRET excess of the Galactic diffuse gamma rays and in disagreement with earlier analysis. The remaining soft contribution of the EGB is shown to resemble the spectra of the observed point sources in our Galaxy.
\end{abstract}

Key words. diffuse radiation - dark matter - gamma rays: observations

\section{Introduction}

In a previous paper (De Boer et al. 2005a, in the following called Paper I), we discussed the possibility to explain the observed excess in the EGRET data (Hunter et al. 1997) in the diffuse Galactic gamma rays above $1 \mathrm{GeV}$ as a dark matter annihilation (DMA) signal. The excess was found to be compatible with a DMA signal from Weakly Interacting Massive Particles (WIMPs) in a mass range from $\sim 50$ to $100 \mathrm{GeV}$. All properties are perfectly consistent with the WIMP being the expected neutralino of the Minimal Supersymmetric extension of the Standard Model (MSSM) of particle interactions (De Boer et al. 2006a). In the analysis presented in Paper I only the spectral shapes for the cosmic ray induced contribution and the DMA contribution to gamma rays are used. Thus no absolute normalization of the cosmic ray induced contribution from Galactic propagation models is needed and also the overall experimental errors largely cancel in the relative contribution of various sky directions which determine the distribution of dark matter. The EGRET excess indeed traces the DM halo, as proven by reconstructing the Galactic rotation curve from it and explaining for the first time its peculiar shape. Arguments against the DMA interpretation of the EGRET excess concerning a too high antiproton flux are based on simplistic propagation models with isotropic propagation (Bergström et al. 2006). These arguments can be invalidated by more realistic propagation models based on anisotropic propagation (De Boer et al. 2006b). Independent evidence in favor of the DMA interpretation of the EGRET excess comes from: a) the $\mathrm{N}$-body simulation of the Canis Major dwarf galaxy as a progenitor of the Monoceros stream, which yields a ring of Dark Matter at 13 $\mathrm{kpc}$ from the Galactic center (Penarrubia et al. 2005) in perfect agreement with the EGRET excess in Paper I; b) the anomalous gas flaring in the outer Galaxy, as was recently shown by
Kalberla et al. (2007) and c) the anomalous change of slope in the rotation curve (see Paper I).

The flux of diffuse gamma rays has a small component from the extragalactic background (EGB). The origin of these gamma rays are other galaxies which might have the same production mechanisms for gamma rays as our Galaxy, or quite different sources like Active Galactic Nuclei (AGN), quasars or blazars. Since the nature of the extragalactic objects is unknown it is difficult to make any predictions about the shape or the absolute value of this background component. However, under the assumption of an isotropic extragalactic background it is possible to determine the EGB experimentally, as done first in a pioneering paper from Sreekumar et al. (1998). They determined the EGB by plotting the observed flux in many different sky direction versus an expected Galactic flux from a Galactic Model (GM). Extrapolating the observed flux to zero Galactic flux yields the EGB. Such an analysis was later repeated with more advanced Galactic models (Strong et al. 2004a), which yielded a lower EGB. None of the GMs used so far includes contributions from DMA, but it is exactly DMA which may provide a large fraction of the diffuse Galactic gamma rays, as demonstrated in Paper I and the DMA is then expected to contribute to the EGB as well.

Subtracting the GM contribution from the observed flux by including or excluding DMA in the GM changes the results. E.g. a recent analysis of the EGB, based on a GM without DMA Strong et al. (2004a), could explain the high energy tail of the DMA with a WIMP mass of $515_{-75}^{+110} \mathrm{GeV}$ (Elsässer et al. 2005). This mass is incompatible with the Galactic DMA signal, which requires a mass below $100 \mathrm{GeV}$ as discussed above and pointed out in a comment by De Boer et al. (2005b). It is the purpose of this paper to show that if DMA is taken into account in the GM, the best fitted WIMP mass in the EGB is reduced and is compatible with the WIMP mass of the Galactic excess. In addition, if the characteristic high energy bump of the EGB is attributed to 
DMA, the shape of the remaining soft part can be determined. The soft part of the EGB was found to resemble the spectra of the observed point sources in our Galaxy.

\section{Method to determine the extragalactic background}

The main concept of the EGB determination is the following (Sreekumar et al. 1998): first the sky map of the diffuse gamma rays is divided into several regions to get high and low fluxes, but the EGB is assumed to be isotropic, so each region will have the same flux from the EGB. For these regions the measured gamma flux is plotted against the flux of a certain GM. If the model is good, one expects a linear dependence with a gradient of one. With an extrapolation to zero Galactic flux from the GM one obtains the EGB from the $y$-axis intercept. This procedure can be repeated for different energy intervals, thus obtaining the EGB as a function of energy.

The uncertainties induced by the GMs can be reduced by not relying on the absolute fluxes, but only fitting the shape of the Galactic flux predicted by the model, thus leaving the absolute normalization of the Galactic flux a free parameter for each sky direction. This eliminates the dependence on fluctuations in gas densities, the interstellar radiation field and the cosmic ray densities. The total gamma ray flux in a given direction $\Psi$ can be written as a sum of the cosmic ray induced gamma rays interacting with the gas, as described by GMs, as well as contributions from DMA and EGB:

$\Phi_{\mathrm{obs}}(\Psi)=f \cdot \Phi_{\mathrm{GM}}(\Psi)+g \cdot \Phi_{\mathrm{DM}}(\Psi)+\Phi_{\mathrm{EGB}}$.

Here $f$ and $g$ are the free normalization factors for the GM- and DM-contribution, respectively, if only the shape is fitted. The procedure we used for the EGB determination can be summarized as follows: first the spectral shape of the cosmic ray induced gamma rays and the shape from DMA have to be chosen. The shape of the GM contribution is taken from the publicly available GALPROP model (Strong \& Moskalenko 1998; Moskalenko et al. 1998; Strong et al. 2000), which is the most detailed GM available. We used the so called conventional GALPROP model (CM), which reproduces the locally measured fluxes of electrons and protons as well as the secondary to primary ratio $\mathrm{B} / \mathrm{C}$. But it fails to describe the spectrum of diffuse gamma rays, if one assumes the local CR spectra to be representative for the Galaxy. This should be a good hypothesis, since the diffusion time is short compared to the energy loss time. The hypothesis is supported by the fact that one can fit the gamma ray spectrum in all sky directions with the locally measure cosmic ray spectrum. Since we use only one normalization constant per sky region for the Galactic background, the ratios of the different contributions (decay of $\pi^{0}$, inverse compton and bremsstrahlung) are estimated from data, as implemented in the GALPROP package.

The shape of the DMA is taken to correspond to a $60 \mathrm{GeV}$ WIMP, as presented in Paper I. In the EGB determination method used in this analysis the normalization factors $f$ and $g$ of the Galactic background and the signal are obtained from a spectral fit of the two contributions to the EGRET data. The normalization factors are allowed to be different for each direction within an assumed uncertainty, as expected from uncertainties in the gas densities and from a possible substructure of the dark matter.

The scaling parameters $f$ and $g$ of the GM and the DMA contribution depend on the EGB, since a larger extragalactic component leads to a smaller galactic one and vice versa. Therefore one has to determine the EGB iteratively. The following algorithm was used:

1. Set the extragalactic background to zero.

2. Determine the direction dependent scaling factors $f^{i, j}$ and $g^{i, j}$ for $i_{\max } \times j_{\max }=18 \times 3=54$ independent sky regions from a fit to the spectral shape of the observed gamma ray flux in the region with indices $i, j$, as done in Paper I. The indices $i$ and $j$ denote the longitude and latitude interval, respectively. In this paper we use 18 equidistant intervals in galactic longitude and 3 equidistant intervals from 10 to 90 degrees. We also tried 6 intervals for the latitude to take positive and negative values separately. The Galactic plane is excluded to eliminate effects from spiral arms and other DM structures in the disc, as observed in Paper I. The scaling factors $f^{i, j}$ are constrained to a value of $f=1$ within the normalization uncertainty $\sigma_{f}$ by adding a term $(f-1)^{2} / \sigma_{f}^{2}$ to the $\chi^{2}$. Similarly the scaling of the DMA is constrained by adding a term $(g-1)^{2} / \sigma_{g}^{2}$. Numerical values of these scaling errors will be discussed later. The scaling factors $f^{i, j}$ and $g^{i, j}$ have been determined from the fit to the spectral shape of the gamma rays in the region $(i, j)$, as in Paper $\mathrm{I}$.

3. Choose an energy interval. The observed flux is plotted against the Galactic flux $f \cdot \Phi_{\mathrm{GM}}+g \cdot \Phi_{\mathrm{DM}}$ for the sky regions introduced earlier. Clearly $\Phi_{\mathrm{EGB}}=\Phi_{\mathrm{ob}}$ for $f \cdot \Phi_{\mathrm{GM}}+g \cdot \Phi_{\mathrm{DM}}=$ 0 , i.e. the EGB can be obtained from the $y$-axis intercept of a linear fit (Eq. (1)) through these points. Since the fit function contains the unknown $\Phi_{\mathrm{EGB}}$, the final solution can only be found by an iterative procedure, described by the next steps.

4. Go back to step 3 and repeat the procedure for the next energy interval of gamma rays in order to obtain the complete EGB spectrum.

5. Go back to step 2, unless the EGB has not changed within a given accuracy.

\section{Results}

\subsection{EGB determination}

In Fig. 1 the linear fits of the correlation between the observed flux and the GM flux are shown for 3 different energy ranges following the description of the previous section. For comparison also the EGB using GALPROP without GM scaling and without DMA contribution is shown on the right hand side. Clearly the slopes and offsets representing the EGB are significantly different. For a model including DMA the slope is close to one, as expected for a correct model. Nevertheless the model without DM has almost the same intercepts and therefore the same EGB. At high energies the difference of the extrapolation originates from the DMA contribution and the GM scaling, while at lower energies only the GM scaling effects the result.

After repeating these linear fits for the 8 highest EGRET energy intervals (above $70 \mathrm{MeV}$ ) one obtains the spectral shape of the extragalactic background, as shown in Fig. 2. The EGBs for the CM are calculated with the four different combinations: with/without GM scaling for each sky map region and with/without DMA signal.

The following points are noteworthy:

- A comparison with previous EGB determinations, indicated by the hatched areas (upper one from Sreekumar et al. 1998, lower one from Strong et al. 2004a) shows that our results are in reasonable agreement with the ones from Strong et al. (2004a). This is not surprising since the lower hatched 

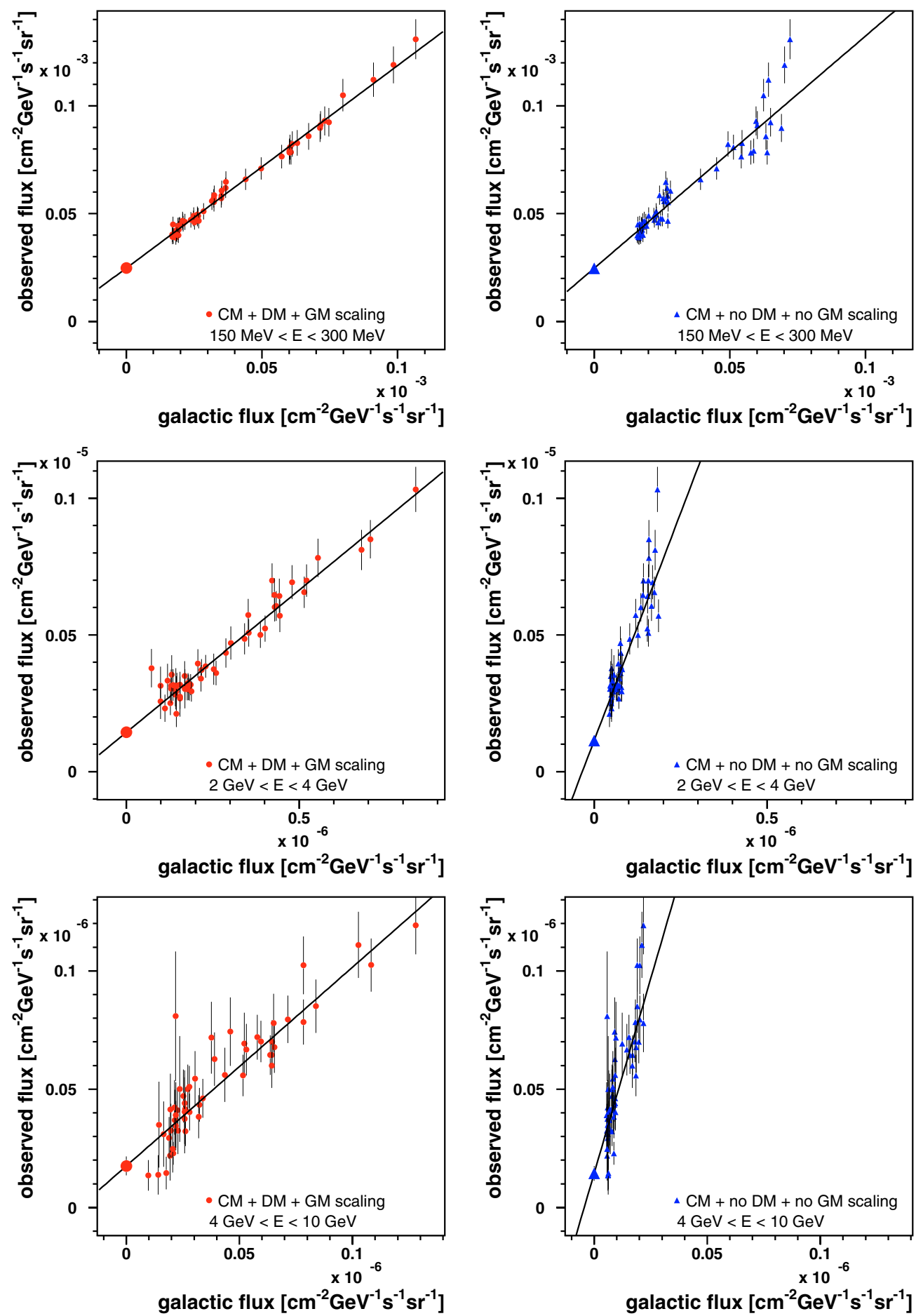

Fig. 1. A plot of the observed flux of diffuse gamma rays (with statistical errors only) versus the Galactic flux $\left(=\Phi_{\mathrm{GM}}+\Phi_{\mathrm{DM}}\right.$ ) for three of the ten EGRET energies (150 to $300 \mathrm{MeV}$ (top), 2000-4000 MeV (middle) and 4 to $10 \mathrm{GeV}$ (bottom). On the left hand side a DMA contribution corresponding to a WIMP mass of $\sim 60 \mathrm{GeV}$ was taken into account and the GM normalization was left as a free parameter, while on the right hand side the EGB was determined in the simplest way, i.e. the Galactic flux is directly taken from GALPROP without scaling and without DMA contribution. Note the strong change of slope at high energies caused by the DMA contribution. The extrapolated point (large symbol) at the Galactic flux $\Phi_{\mathrm{GM}}+\Phi_{\mathrm{DM}}=0$ is the EGB.

area has also been determined with the GALPROP package, while the upper one is based on a simpler model with an underestimated inverse Compton contribution. The effect of the scaling of the GM contribution increases the EGB, while the DMA decreases the EGB below $3 \mathrm{GeV}$ and enhances it above $3 \mathrm{GeV}$ (see left panel of Fig. 2). Therefore the region around $3 \mathrm{GeV}$ is quite stable. The net result is a decrease of the high energy tail and enhancing the structure around $3 \mathrm{GeV}$. Note that both previous determinations have not been done with a region dependent GM scaling, but a scaling for each energy interval to take care of the imperfect description of the spectral gamma ray shape. This scaling is taken to be the same for all regions and leads to the difference between the methods. 

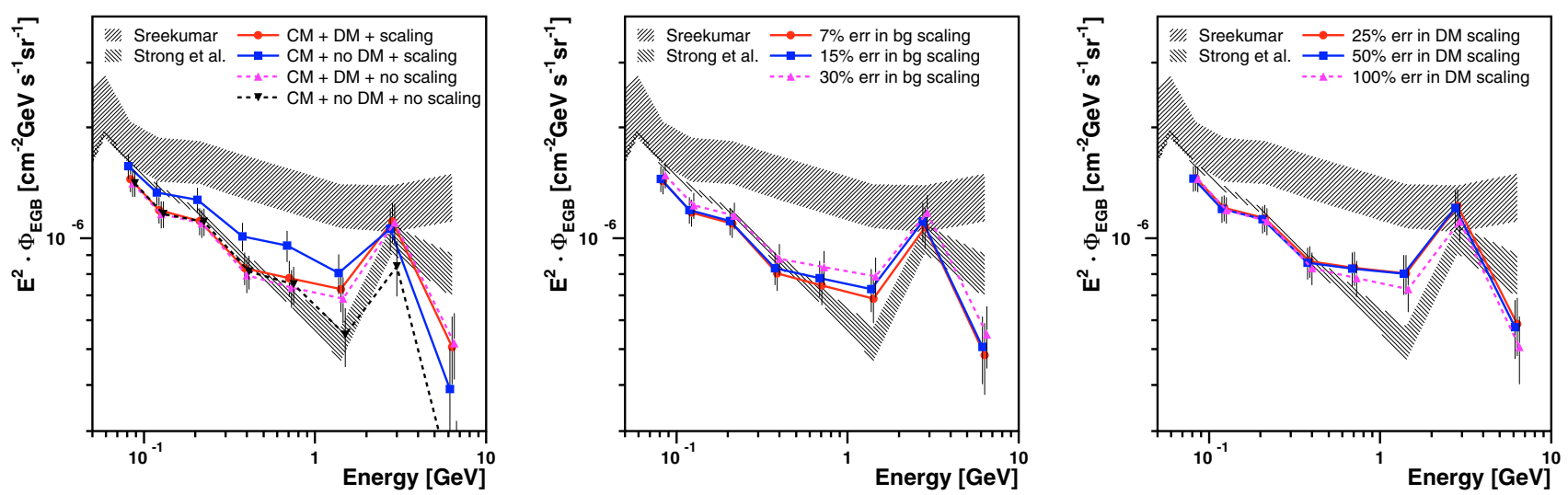

Fig. 2. Left: the EGB with four different curves corresponding to different methods (with/without DMA contribution and with/without GM scaling. Only statistical errors are plotted. In the middle and right hand panel the dependence of the EGBs on the scaling errors $\sigma_{f}$ and $\sigma_{g}$ are shown. The two shaded bands are the EGB determined by Sreekumar et al. (1998) (highest band, including systematic errors) and Strong et al. (2004a) (lower band, only statistical errors).

Table 1. The EGB flux ( $E^{2} \Phi_{\mathrm{EGB}}$ and $\Phi_{\mathrm{EGB}}$ ) for different energies with the statistical errors in brackets. For the energy $E$ of an interval the geometric average $\sqrt{E_{\max } \cdot E_{\min }}$ is taken. In Cols. 4 to 6 the systematic uncertainties on the spectral shape from the data, GM uncertainty and DM uncertainty are shown. They were determined by half of the variation of the EGB caused by changing the EGRET data by $\pm 15 \%$, which is the estimated EGRET absolute normalization error and by varying the GM and the DM scaling errors as indicated in Fig. 2. Note that increasing the EGRET data will also increase the prediction of the GM, since only the shape is fitted, so the effect of the normalization uncertainty is reduced. The total systematic uncertainty in the last column includes a point to point error of the EGRET experiment which is taken from Paper I to be $7 \%$. All errors are added in quadrature.

\begin{tabular}{ccccccc}
\hline \hline Energy $[\mathrm{MeV}]$ & $E^{2} \cdot \Phi_{\mathrm{EGB}}\left[\mathrm{GeV} \mathrm{cm} \mathrm{s}^{-1} \mathrm{sr}^{-1}\right]$ & $\Phi_{\mathrm{EGB}}\left[\mathrm{GeV}^{-1} \mathrm{~cm}^{-2} \mathrm{~s}^{-1} \mathrm{sr}^{-1}\right]$ & $\sigma_{\mathrm{EGRET}}$ & $\sigma_{\mathrm{GM}}$ & $\sigma_{\mathrm{DM}}$ & $\sigma_{\mathrm{tot}}$ \\
\hline $70 \ldots 100$ & $1.45(5) \times 10^{-6}$ & $2.07(8) \times 10^{-4}$ & $0.7 \%$ & $1.1 \%$ & $1.0 \%$ & $7.2 \%$ \\
$100 \ldots 150$ & $1.19(5) \times 10^{-6}$ & $7.96(34) \times 10^{-5}$ & $0.8 \%$ & $0.6 \%$ & $0.7 \%$ & $7.1 \%$ \\
$150 \ldots 300$ & $1.11(5) \times 10^{-6}$ & $2.47(12) \times 10^{-5}$ & $1.7 \%$ & $0.4 \%$ & $0.3 \%$ & $7.2 \%$ \\
$300 \ldots 500$ & $0.83(6) \times 10^{-6}$ & $5.53(41) \times 10^{-6}$ & $3.6 \%$ & $1.7 \%$ & $0.8 \%$ & $8.1 \%$ \\
$500 \ldots 1000$ & $0.78(7) \times 10^{-6}$ & $1.56(14) \times 10^{-6}$ & $1.0 \%$ & $2.8 \%$ & $1.8 \%$ & $7.8 \%$ \\
$1000 \ldots 2000$ & $0.73(8) \times 10^{-6}$ & $3.64(42) \times 10^{-7}$ & $4.2 \%$ & $4.1 \%$ & $3.5 \%$ & $9.8 \%$ \\
$2000 \ldots 4000$ & $1.11(11) \times 10^{-6}$ & $1.39(14) \times 10^{-7}$ & $8.6 \%$ & $1.7 \%$ & $3.4 \%$ & $11.7 \%$ \\
$4000 \ldots 10000$ & $0.51(10) \times 10^{-6}$ & $1.26(25) \times 10^{-8}$ & $12.7 \%$ & $3.6 \%$ & $5.7 \%$ & $16.1 \%$ \\
\hline
\end{tabular}

- In the middle panel of Fig. 2 the EGB is shown for three values of the normalization error, namely $\sigma_{f}-0.07,0.15,0.3$, respectively. The EGB increases continuously for an increasing uncertainty of the scaling. This effect is due to the correlation between the DMA signal, the GM and the EGB, which can be understood as follows: let us assume that the spectral fit of the diffuse gamma rays prefers a smaller GM contribution, i.e. a smaller $f^{i, j}$. This shifts the fitted line in a correlation plot like Fig. 1 to the left which leads to a larger $y$-axis intercept, i.e. a larger EGB. Consequently in the next iteration step a even smaller GM contribution is needed which causes the algorithm to converge at a larger EGB. A similar effect happens with the uncertainty in the DMA scaling, but with an opposite sign, as shown in the right panel and in Table 1. The overall spectral shape of the EGB is only weakly dependent on these systematic errors, so the characteristic bump above $1 \mathrm{GeV}$ is hardly affected.

\subsection{Anisotropy of the EGB}

The EGB in the previous section was assumed to be isotropic. To test this hypothesis the EGB has been determined for various subsections of the sky. The Galactic plane (latitudes smaller than $10^{\circ}$ ) has been excluded in all cases, since in this region the EGB is much lower than the Galactic gamma rays and the diffuse gamma rays strongly depend on inhomogeneous structures, both in the baryonic and dark matter. In Fig. 3 the EGB is shown for the hemispheres with positive or negative latitude as well as for positive or negative longitudes. The different hemispheres for positive and negative longitudes show good agreement with each other, while the hemispheres with positive and negative latitudes have a difference of $\sim 15 \%$. A similar anisotropy was also observed by Sreekumar et al. (1997) and it shows that the EGB is not perfectly isotropic, if one assumes it is not an experimental effect. This is not excluded, since the detector efficiency changed in time and the exposure times were not equal for all sky directions. An alternative reason for the observed anisotropy could be the fact, that the earth is not located exactly in the galactic plane but a few tens of pc above the plane. Or equivalently the Milky Way baryonic and/or DM contribution is not perfect symmetric with respect to the plane, but in the GM it is assumed to be symmetric. Therefore it is important to average the regions for positive and negative latitudes. It should be pointed out that for both hemispheres the EGB spectra show the characteristic feature of the high energy bump.

\subsection{Contribution of DMA in the EGB}

The bump in the EGB at $3 \mathrm{GeV}$ is indicative of an extragalactic DMA contribution. This characteristic feature does not depend on any of the uncertainties discussed in the previous sections. If this feature is fitted with a DMA contribution for a $60 \mathrm{GeV}$ 

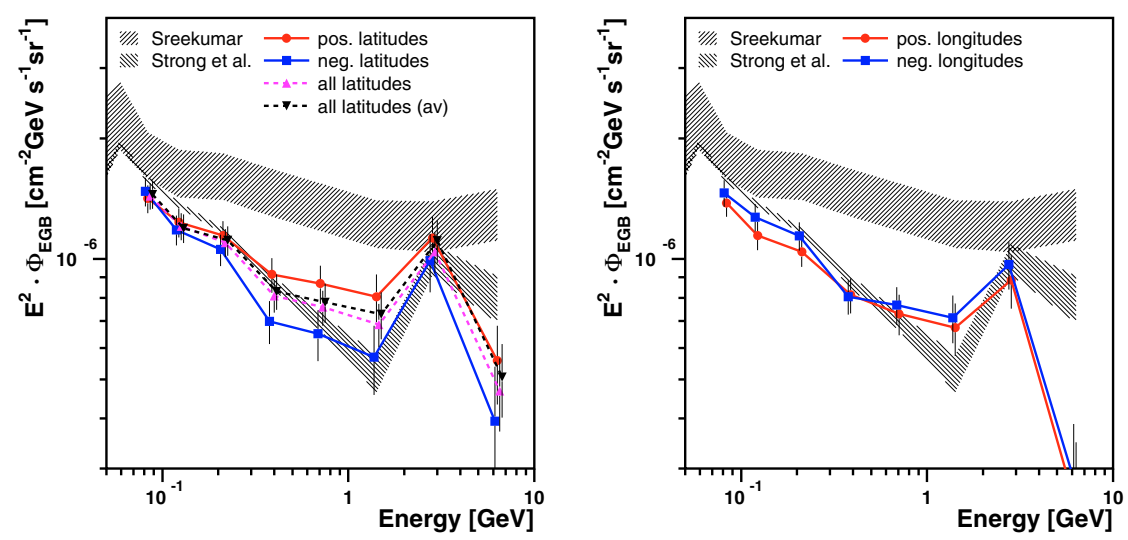

Fig. 3. Spectrum of the extragalactic background determined for the conventional model with GM scaling and DMA for different hemispheres. The systematic point to point error is not included since it is common to all sky regions, so only the statistical errors are plotted.

Table 2. Parameters of the fit to the shape of the EGB with a single power law and a double power law (see Eq. (2), in which the second term in the denominator (with $\gamma_{2}$ ) should be ignored for the single power law). For the double power law the DMA was included in the fit using the shape of the DMA spectrum of Paper I.

\begin{tabular}{ccc}
\hline \hline Parameter & single power & double power \\
\hline$\Phi_{0}\left[\mathrm{GeV} \mathrm{cm} \mathrm{cm}^{-2} \mathrm{~s}^{-1} \mathrm{sr}^{-1}\right]$ & $(4.5 \pm 0.2) \times 10^{-7}$ & $(5.3 \pm 0.6) \times 10^{-7}$ \\
$E_{0}[\mathrm{GeV}]$ & 0.5 & 0.5 \\
$\gamma_{1}$ & $-0.21 \pm 0.04$ & $-0.19 \pm 0.09$ \\
$\gamma_{2}$ & - & $-1.74 \pm 0.53$ \\
\hline$\chi^{2} /$ d.o.f. & $11.7 / 6$ & $5.7 / 4$ \\
probability & $6.9 \times 10^{-2}$ & $22.4 \times 10^{-2}$ \\
\hline
\end{tabular}

Wimp, as needed to describe the Galactic EGRET excess, then the remaining part of the spectrum is the contribution of all extragalactic sources except the DMA contribution. This remaining part can be parametrized by a double power law, typically used for point sources:

$E^{2} \cdot \Phi_{\mathrm{EGB}}=\frac{2 \Phi_{0}}{\left(\frac{E}{E_{0}}\right)^{-\gamma_{1}}+\left(\frac{E}{E_{0}}\right)^{-\gamma_{2}}}$.

The best fit parameters of a combined fit of DMA plus EGB $_{\text {non-DM }}$ are given in Table 2 and the fits are compared with the data in Fig. 4. There is a strong correlation between the two slopes in the double power law and the transition energy $E_{0}$, i.e. the EGB at low energies can be fitted either by the contribution below or above the break. So we fixed the transition energy $E_{0}$ to $0.5 \mathrm{GeV}$ which hardly changes the total $\chi^{2}$. The model including DMA has a larger probability than the model without DM (using a single power law, see Table 2), because of the two highest energy points. A WIMP mass of $60 \mathrm{GeV}$ provides certainly a possibility to explain the bump in the EGB and is compatible with the excess in the Galactic data in contrast to the WIMP mass determination by Elsässer et al. (2005), as discussed in the introduction. Note that the statistical significance for the single high point is not overwhelming, as shown by the rather small difference in probabilities of the single and double power law in Table 2. Therefore other explanations, like additional contributions from AGN's or blazars are certainly not excluded. However, the fact that the bump just occurs at the energy interval, where also the Galactic excess has a maximum, strengthens the DMA interpretation. In this case the dominant contribution to the EGB flux of diffuse gamma rays at high energies originated from DMA (see Fig. 4), just like it is the case for the Galactic flux, which

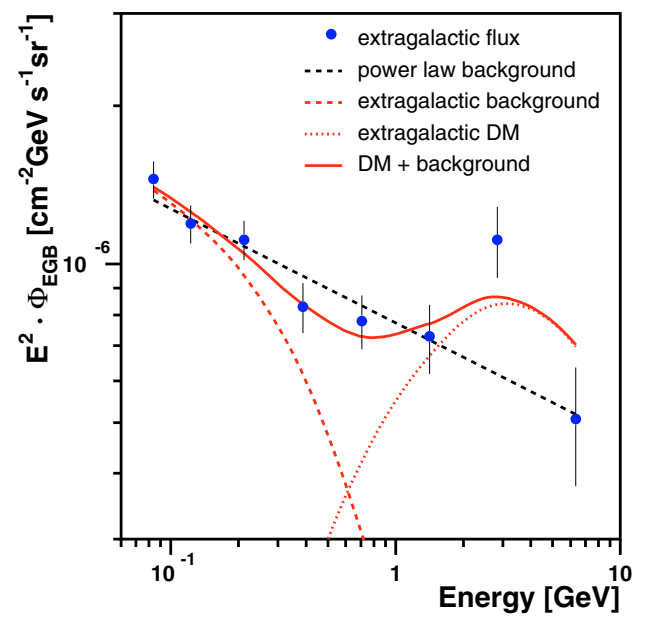

Fig. 4. Fits to the extragalactic background with a single power law (dashed black) and a double power law (dashed, red) plus a DMA contribution (dotted, red). In this plot the complete error is included.

is also dominated by DMA for energies above $2 \mathrm{GeV}$. The remaining soft contribution of the EGB resembles the spectra of the many point sources in our Galaxy, as shown in the Appendix of Paper I.

\section{Conclusion}

A new iterative method for the determination of the isotropic EGB has been presented, relying mainly on the spectral shape of the gamma rays from a Galactic Model. The influence of the Galactic DMA on the EGB was studied as well. The newly determined EGB shows a characteristic bump around $3 \mathrm{GeV}$, i.e. at the same position as the Galactic EGRET excess. Therefore this bump can be fitted with the same WIMP mass as determined in Paper I from the Galactic excess, which is much lower than the WIMP mass around $500 \mathrm{GeV}$ found by Elsässer et al. (2005). This bump is insensitive to all possible uncertainties considered and is therefore real, although its statistical significance is less than $2 \sigma$, since a single power law gives already a probability of $7 \%$. If nevertheless a DMA contribution is fitted, the EGB flux above $1 \mathrm{GeV}$ is dominated by the extragalactic DMA, as shown in Fig. 4 and the remaining soft contribution in the EGB can be described by a power law reminiscent of the spectra of the typical point sources in our Galaxy (see Paper I). 
Acknowledgements. We thank O. Reimer for a meticulous reading of the manuscript and healthy criticism. This work was supported by BMBF (Bundesministerium für Bildung und Forschung) via the DLR (Deutsches Zentrum für Luft- und Raumfahrt).

\section{References}

Bergström, L., Edsjö, J., Gustafsson, M., \& Salati, P. 2006, JCAP, 0605, 006 de Boer, W., Sander, C., Zhukov, V., Gladyshev, A. V., \& Kazakov, D. I. 2005a, A\&A, 444, 51 (Paper I)

de Boer, W., Sander, C., Zhukov, V., Gladyshev, A. V., \& Kazakov, D. I. 2005b, Phys. Rev. Lett. 95, 209001

de Boer, W., Sander, C., Zhukov, V., Gladyshev, A. V., \& Kazakov, D. I. 2006a, Phys. Lett. B, 636, 13

de Boer, W., Gebauer, I., Sander, C., Weber, M., \& Zhukov, V. 2006b, to be published in the Proc. of the 7th UCLA Symposium on Sources and Detection of Dark Matter and Dark Energy in the Universe, Marina del Rey, [arXiv:astro-ph/0612462]
Elsässer, D., \& Mannheim, K. 2005, Phys. Rev. Lett. 94, 171302

Esposito, J. A., Bertsch, D. L., Chen, A. W., et al. 1999, ApJ, 123, 203

Hunter, S. D., Bertsch, D. L., Catelli, J. R., et al. 1997, ApJ, 481, 205

Kalberla, P. M. W., Dedes, L., Kerp, J., \& Haud, U. 2007, Dark matter in the Milky Way II. the HI gas distribution as a tracer of the gravitational potential, ApJ, submitted

Moskalenko, I. V., Strong, A. W., \& Reimer, O. 1998, A\&A, 338, L75

Penarrubia, J., et al. [SDSS Collaboration] 2005, ApJ, 626, 128

Sreekumar, P., Stecker, F. W., \& Kappadath, S. C. 1997, AIP Conf. Proc., 410, Proceedings of the Fourth Compton Symposium, 344

Sreekumar, P., et al. [EGRET Collaboration] 1998, ApJ, 494, 523

Strong, A. W., \& Moskalenko, I. V. 1998, ApJ, 509, 212

Strong, A. W., Moskalenko, I. V., \& Reimer, O. 2000, ApJ, 537, 763 [Erratum-ibid. 541, 1109] [arXiv:astro-ph/9811296]; Details on the latest GALPROP versions can be found at

http://www.mpe.mpg.de/ aws/propagate.html

Strong, A. W., Moskalenko, I. V., \& Reimer, O. 2004a, ApJ, 613, 956

Thompson, D. J., Bertsch, D. L., Fichtel, C. E., et al. 1987, IEEE Trans. Nucl. Sci. 34,36 